\title{
RISKGATE y operaciones en minas de carbón en Australia
}

\section{RISKGATE and Australian coal operations}

\author{
Philipp Kirsch', Jill Harris', Darren Sprott ${ }^{2}$ Ángela Calderón'
}

1 Minerals Industry Safety and Health Centre. Sustainable Minerals Institute. University of Queensland. Sir James Foots Building. Brisbane, Queensland Australia 4072.

2 Design Solutions Pty. Ltd. 4 Mankina Court PO Box 1199 Buddina, Queensland, Australia 4575.

Recibido: 24-03-14

Aceptado: 18-05-14

\section{Correspondencia}

Philipp Kirsch

Minerals Industry Safety and Health Centre

Sustainable Minerals Institute

University of Queensland

Sir James Foots Building

Brisbane, Queensland Australia 4072

Correo electrónico p.kirsch@uq.edu.au

Tfno: 0433998255

Resumen

El principal programa de investigación de la Asociación de Carbón en Australia (ACARP), RISKGATE ha completado tres años de conocimiento en la captura y el desarrollo del sistema. El cuerpo de conocimiento del manejo de riesgos de neumáticos, colisiones, incendios, aislamiento, estratos de suelo en las minas subterráneas, suelo en minas de cielo abierto, explosiones, explosivos en minas subterráneas, explosivos en minas de cielo abierto, trabajos manuales, resbalones/tropiezos/caídas fue lanzada en diciembre del 2012. Recientemente, el proyecto a adicionado al cuerpo de conocimiento temas relacionados a escapes de gas espontaneo, fallas en la extracción de carbón, interface entre la interacción máquina-humano, depósito de escoria, higiene ocupacional y escape de cuerpos de agua a los originales 11 tópicos. En el 2014, los planes del proyecto (pendiente a la aprobación a la fundación de ACARP) es al enfoque en problemas relacionados con salud ocupacional.

RISKGATE provee un ambiente en la captura de conocimiento y reciprocidad en un mundo de innovación e intercambio de prácticas actuales a través de la industria en la identificación, evaluación y manejo de riesgo. En la captura del conocimiento operacional por medio de expertos industriales, RISKGATE provee memoria corporativa acumulativa en un momento de alta rotación del personal en la industria del carbón.

Este artículo presenta una visión en conjunto de los primeros diecisiete tópicos, estructura de los tópicos y contraste de relaciones internas entre los tópicos. La segunda parte del articulo discute algunos primeros pasos que las compañías están tomando para integrar RISKGATE en estas operaciones; y concluye con algunos ideas en donde RISKGATE puede ir en un futuro.

Med Segur Trab (Internet) 2014; 60 (235) 290-303

Palabras claves: Manejo de riesgos, memoria corporativa, ACARP, eficacia en controles, trabajo seguro en minas de carbón. 


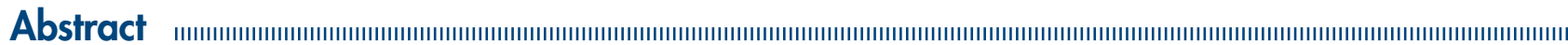

The major Australian Coal Association Research Program (ACARP) project, RISKGATE has now completed three years of knowledge capture and system development. The body of knowledge for risk management of tyres, collisions, fires, isolation, strata underground, ground control open cut, explosions, explosives underground, explosives open cut, manual tasks and slips/trips/falls was launched in December 2012. Recently, the project added knowledge about outbursts, coal bumps and bursts, human-machine interface, tailings dams, occupational hygiene and inrush to the original 11 topics. In 2014, the project plans (pending ACARP funding approval) to focus on issues around Fitness for Work.

RISKGATE provides an environment for knowledge capture and knowledge exchange to drive innovation and cross industry sharing of current practice in the identification, assessment and management of risk. By capturing operational knowledge from industry experts, RISKGATE provides a cumulative corporate memory at a time of high personnel turnover in the coal industry. RISKGATE is the largest single ACARP Occupational Health and Safety (OHS) initiative to date.

This paper presents an overview of the first seventeen topics, topic structures, and contrasts and interrelationships between topics. The second part of the paper discusses some early steps that companies are taking to integrate RISKGATE into their operations; and conclude with some thoughts on where RISKGATE can go in the future.

Med Segur Trab (Internet) 2014; 60 (235) 290-303

Key words: Risk management, corporate memory, ACARP, control effectiveness, work safety in coal 


\section{INTRODUCTION}

RISKGATE es una herramienta basado en la web (RISKGATE, 2013) la cual provee clara, actualizada y contiene una práctica lista de chequeo en el control de los 17 eventos específicos con mayor prioridad de eventos no deseados en las operaciones de carbón en Australia. Basado en el sistema interactivo "análisis de corbatín" (BTA) asistiendo en la implementación en operaciones seguras, cada tópico en RISKGATE y cada análisis de corbatín está centrado en específicos eventos de iniciativa. La canalización de las causas y consecuencias a través de los eventos de iniciativa mantiene la información concisa, intuitiva y selectiva. Usuarios pueden generar listas de chequeos que puedan comunicar los gerentes y los ingenieros rápidamente y con acceso relevante para las prácticas de controles actuales en la industria, que se pongan en consideración para sus propios sitios de trabajo. Estas listas de chequeo están designadas como referentes en prácticas actuales que puedan asistir con la evaluación de riesgos, auditoría, investigación de accidente, y entrenamiento. Las guías para usuarios y otros materiales que asistan en la implementación de este cuerpo de conocimiento en las operaciones de minería en carbón se pueden obtener por medio del autor, o en línea en la página web (RISKGATE, 2013).

RISKGATE está financiado por el Programa de Investigación de la Asociación de carbón en Australia (ACARP); manejada e implementada por la Universidad de Queensland; y cada uno de los miles de controles específicos implementados en el sistema de RISKGATE ha sido considerado y evaluado por los expertos de industria por las compañías líderes de minería en Australia. RISKGATE ha sido creado por medio del conocimiento de expertos de industria reunido a través de talleres específicos en los tópicos de investigación (Kirsch et al., 2012, 2013ab; Worden et al., 2013). Los tópicos que ya han sido completados incluye: incendios (Harris et al., 2012), controles a las capas de suelo en minas subterráneos (Kirsch et al., 2013c), controles de suelo en minas de cielo abierto, colisiones, neumáticos, aislamiento, explosiones (Kirsch et al., 2013c), explosivos (Harris et al., 2013), trabajos manuales, y resbalones/tropiezos/caídas (Lynas et al., 2014, in press). El propósito de RISKGATE es no evaluar específicamente riesgos en un único lugar, sino que provee una herramienta de soporte en la toma de decisiones y rendimiento, tales como listas de chequeo adaptadas, que puede asistir a usuarios en sus lugares de trabajo en la evaluación de riesgos y manejo de ellos.

\section{RISKGATE 2011-2013}

En respuesta a la solicitud de ACARP, en el 2010 en el Centro de Seguridad Industrial y Salud del Instituto de Minerales (MISCH) de la Universidad de Queensland desarrollo RISKGATE en consulta con representantes seleccionados en la industria de carbón (Kirsch et al., 2012). Es importante destacar que los peligros envueltos en minería de carbón (referidos a los tópicos en RISKGATE), RISKGATE los incluye. En los últimos tres años, este enfoque será expandido a la solicitud de la industria desde los 12 primeros originales tópicos a los actuales 17 tópicos que se describen en este escrito (Figura 1). Cada tópico en RISKGATE está enfocado en las actividades industriales de carbón (minería, procesamiento, transporte y almacenamiento) en ambos ambientes de minería, a cielo abierto y subterráneo. El enfoque incluye sitios mineros, áreas abiertas e infraestructura minera (e.g. móvil, planta fija, equipo de campo, edificios y transporte, incluyendo carretera y ferrocarril); y todos los aspectos en el ciclo de vida de la mina desde el diseño hasta el cierre. Los tópicos reconocen que "la pérdida de control" puede resultar en personal lesionado y/o fatalidades, daños de equipos, perdida en la producción, perdida de reputación y daño ambiental. Sin embargo, la prioridad de RISKGATE está enfocada primordialmente en la seguridad de los trabajadores. 
2011

2012

2013

2014

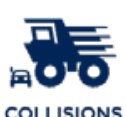

COLLISIONS
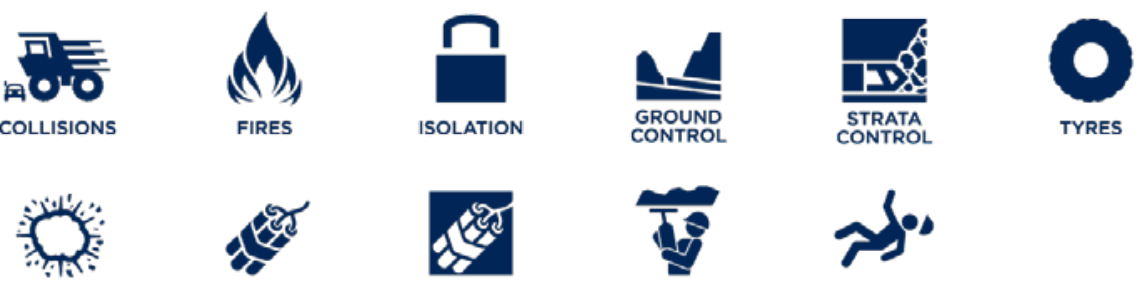

EXPLOSIONS
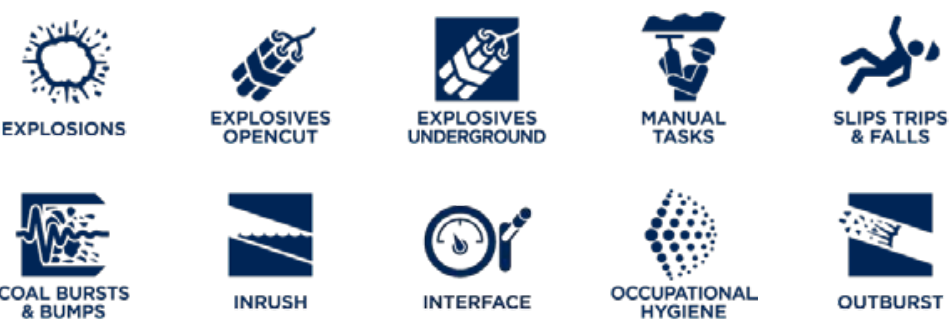

Salud

Ocupacional

Figura 1. RISKGATE tópicos y desarrollo del programa 2011-2014

\section{RISKGATE Resumen de los Tópicos}

El sistema de RISKGATE aplica a una estructura estándar a través del cuerpo de conocimiento. Cada tópico está definido en términos en lo que se ha considerado, y también en términos en áreas que han sido excluidos. En cada tópico, el desarrollo de la definición provee términos de referencia para cada evento individual de iniciativa. La intención es que cada uno de los usuarios puede entender como ha sido estructurado el conocimiento. Por ejemplo, un usuario de RISKGATE conduce una investigación de un incidente, este podría empezar con la información acerca de las consecuencias que tiene un evento no deseado (i.e. información encontrada en la parte derecha del corbatín), mientras un usuario conduce una evaluación de riesgos enfocándose en varias causas y controles preventivos de un evento (i.e. información que se encuentra al lado izquierdo del corbatín). Las definiciones de los tópicos individuales están resumidas a continuación:

- INCENDIOS relaciona la indeseada o inesperada combinación de fuente de combustible y fuente de ignición que resulte en incendio. Este genera controles que se enfocan en cuatro contextos relacionados en minería: Incendio en una planta móvil y equipo de campo, Incendio en planta fija e infraestructura, Incendio en el medio ambiente, Incendio en las pilas de almacenamiento. Fuentes de combustible es cualquier material inflamable incluyendo líquidos inflamables (i.e. gasolina, lubricante, fluido del transformador, aceite hidráulico y refrigerante), gases presurizados (LPG, gas natural, acetileno) carbón, otros solidos (e.g. trapos, madera, etc.) y otros fluidos inflamables (e.g. líquidos de limpieza). Fuentes de ignición incluye energía eléctrica, fricción, trabajo caliente, inducción de relámpagos, superficies calientes y otras fuentes diversas. INCENDIOS no aborda explosiones o combustiones espontaneas que suelen abarcar en RISKGATE EXPLOSIONES.

- NEUMATICOS suministra información acerca del manejo y prevención de incidentes y accidentes asociados con el uso de vehículos pesados, usados en la industria y otro tipo de neumáticos, llantas en minas de carbón de cielo abierto y subterráneo. Aquí, el uso de neumáticos incluye todo los aspectos de neumático y manejo del ciclo de vida de llantas - incluyendo la selección, compra, transporte, instalación y remoción, mantenimiento, operación, almacenamiento y disposición final. El enfoque incluye el manejo de neumáticos, llantas en el sitio minero y áreas abiertas cerca al lugar minero.

- AISLAMIENTO proporciona información acerca del manejo y prevención de incidentes y accidentes debido a la falla del proceso de aislamiento in minería de cielo abierto y subterráneo. Las energía relevantes son eléctrica, hidráulica, neumática, gravedad, mecánica, química, termal y radiactiva. Específicamente, 
este tópico abarca el control de energías y establecimiento de barreras para separar energías y la interacción con personas. Cada elemento de proceso de aislamiento (i.e. identificación de la fuente de energía, operación de aislamiento físico del dispositivo, verificación, y cierre) es considerado a través del ciclo completo de vida del proceso de aislamiento en los dispositivos

- CONTROL EN LAS CAPAS DE SUELO suministra información de las fallas debido a la pérdida del control de las capas de suelo en los ambientes de minería subterránea. Hay ocho eventos de iniciativa que han sido identificados en áreas prioritarias donde realza el conocimiento de controles preventivos y de mitigación que podrían dramáticamente reducir la posibilidad y/o severidad de consecuencias. Los eventos de iniciativa son: pérdida de control de la pared lateral, de las carreteras de acceso, pérdida de control en el desarrollo de carreteras, pérdida de control en vías de sobrecarga, comportamiento de túneles, perdida de la estabilidad del sistema en pilas, pérdida de control en la extracción de los bordes de las pilas, pérdida de control de los estratos en la entrada de la mina.

- CONTROL DE SUELO proporciona información en el manejo y prevención de incidentes y accidentes debido a la inestabilidad del suelo en minería a cielo abierto. Inestabilidad de suelo hacen referencia a las paredes del terreno que están afuera del sistema de control de manejo de las capas de suelo (e.g. colapso o desplazamiento). Controles proveen un manejo potencial de eventos no deseados (i.e. eventos de iniciativa) asociados con actividades conducidos en las siguientes locaciones: perdida de estabilidad de la parte superior del terreno y la parte inferior, perdida de la estabilidad de bajas paredes del terreno, perdida de estabilidad de los camiones que transportan el carbón en la minas, perdida de estabilidad de las dragas, perdida de estabilidad de las pilas de almacenamiento, perdida de estabilidad de los cortes que se dan en la mina que le dan forma de escaleras a la mina.

- COLISIONES relacionado a la interacción inesperada entre gente, equipo móvil y fijo, o planta fija; incluye movimientos no controlados de planta movible (donde no otro vehículo o peatones terminen involucrados), resultando en arrastre, deslizamiento, vuelco y caída sobre bordes o huecos. Equipos móviles y de campo (vehículos) están definidos como maquinas autopropulsoras o maquinas que son transportables alrededor de la mina con el fin de realizar funciones básicas (e.g. pesado y livianos vehículos, incluyendo camiones de carga, montacargas, grúas móviles, equipos de movimiento de tierras, dragas, torres de iluminación carros de transporte, 4WD. Plantas fijas hace referencia a la infraestructura no transportable o equipo (e.g. edificios, áreas de parqueo, instalaciones, presas, granjas de tanques, pilas de almacenamiento, líneas de electricidad y redes de transporte). Esto abarca eventos indeseados o interacciones vehículo-vehículo, vehículo-gente (incluye vuelcos), y vehículo-infraestructura que pueden resultar en incidentes singulares o múltiples.

- EXPLOSIONES está definido en una combinación inesperada de combustible y fuente de ignición que resulte en incendio o explosión. Este tópico se enfoca en la minería de carbón y en el proceso de minería a cielo abierto y subterráneo. Esto abarca el potencial de incendio o ignición que se pueda propagar en un incendio a grandes proporciones o explosión con potenciales consecuencias que pueda involucrar múltiples fatalidades, o un extendido daño. Fuentes de combustibles son el carbón o gases inflamables tales como metano, etanol, monóxido de carbono e hidrógeno. Las fuentes de ignición provienen de fuentes de energía que incluye energía eléctrica, fricción, trabajo caliente, inducción de relámpagos, superficies calientes, combustión espontánea y otras fuentes variadas. Esto también incluye incendios presentes en plantas móviles, infraestructura, ambiente natural, pilas de almacenamiento. Control de gas inflamable es posible a través de la práctica de adecuada ventilación, incluyendo drenaje de gas. Acá, los trabajos de minería incluye todas las áreas de la mina 
donde el carbón se está produciendo, áreas que han sido preparado para la extracción de carbón y en aquellas áreas donde ya se ha extraído el carbón.

- EXPLOSIVOS EN MINAS DE CIELO ABIERTO hace referencia a la liberación de energía no planeada de explosivos. Este tópico está enfocado en la sobrecarga en la remoción y la extracción de carbón en operaciones en minería a cielo abierto. También proporciona información de los eventos de voladura tanto dentro como fuera de las zonas de protección y gestión establecidas para la maquinaria y la gente. Este tópico cubre la manufactura, transporte, almacenamiento y uso, también disposición de productos explosivos en la concesión minera. Este material puede ser aplicable a otro (no carbón) en las operaciones de voladura (e.g. minería de metales, cantares, ingeniería civil)

- EXPLOSIVOS EN MINAS SUBTERRANEAS se enfoca en la liberación de energía no planeada de explosivos e incendio no planeado o la consecuente actividad de explosivos, con un enfoque en el uso de explosivos en operaciones de minería subterránea. También se enfoca en los eventos que suceden después de realizarse la voladura tanto dentro como fuera de las zonas de exclusión en las zonas de manejo de las personas y maquinaria. Este tópico cubre el transporte, almacenamiento y disposición de los productos de explosivos en el sitio de la mina. Aspectos de este material puede ser aplicable a otras operaciones en minería subterránea (e.g. minería de metales, construcción de túnel)

- TRABAJOS MANUALES está orientado a los peligros que envuelve los trabajos manuales que conducen a trastornos musculo esquelético. Los trastornos musculo esqueléticos causados por peligros de trabajos manuales incluyen esguinces, distenciones, o desgarros de tejidos (musculo, ligamentos, tendón, discos intervertebrales); fractura por estrés, tendinitis; trastornos vasculares y neuronales. Este tópico considera peligros de trabajos manuales desarrollados por las personas durante la exploración y extracción de carbón en un ambiente subterráneo, también en el transporte de carbón y en el proceso, manipulación del carbón y plantas de preparación.

- RESBALONES, TROPIEZOS Y CAIDAS suministra información en el manejo de peligros asociados con personas que sufren resbalones, tropiezos a nivel de suelo o en escaleras, o plataformas incluyendo estructuras temporales (e.g. construcción de vigas y estructuras para edificios), y el peligro de caer. Este tópico cubre equipos móviles (especialmente acceso y salida) y plantas fijas (incluyendo plantas en la preparación de carbón), también encaja en la actividad de construcción, operaciones y trabajos de mantenimiento, también en la circulación de peatones alrededor del sitio de trabajo. Este tópico incluye la perdida de balance o caída de personas en el mismo nivel, o caída de un nivel a otro. Potenciales consecuencias incluye leves o serias heridas (e.g. esguinces, torceduras de tobillos/rodillas, moretones, fractura de huesos, fractura de cráneo) fatalidades, pérdida de control de cargas o en la realización del trabajo o en la operación de un equipo (e.g. herramientas eléctricas). El enfoque de este tópico se extiende a través de todos los estados de vida de la mina desde la exploración hasta el cierre (incluye el transporte de carbón, en el proceso, manipulación de carbón y preparación de planta). Los controles están dirigidos a situaciones de corto y largo termino. Por ejemplo, diferentes medidas han sido necesarias para la existencia infraestructura a largo plazo que no está conforme con los estándares actuales.

- BULTOS DE CARBON Y ERUPCIONES contribuye a la información en el manejo y prevención de fallas de los estratos de suelo debido a erupciones y pequeños bultos que son usados indistintamente, y pequeños bultos son usados a través de este tópico. El termino bulto describe un violento, frágil, falla dinámica de los estratos de suelo adentro o adyacente a la sección de trabajo debido al estrés afectada por la liberación de energía de forma imprevista. Esto también se puede a la liberación o expulsión de carbón o roca como resultado de la liberación de 
energía. Esto es más comúnmente descrito como una erupción. La ocurrencia de bulto y erupción está directamente relacionado al estrés, y puede potencialmente incrementar con mayor profundidad.

- ESCAPE DE AGUA se trata del incontrolado flujo de líquido, gas u otro material en la operación de mina de carbón que puede resultar en el riesgo inaceptable a la salud y seguridad. Los riesgos son considerados bajo dos eventos de iniciativa: escape de agua en trabajos de superficie y escape de agua en los trabajos realizados en minas subterráneas. Para la caracterización de los peligros asociados con escape de agua requiere la comprensión de un espacio tridimensional que desarrolle e implemente controles efectivos. Dado a la naturaleza del riesgo asociado con irrupción, es importante la continua verificación de los continuos cambios en el ambiente que no impacte en la validad en la evaluación del riesgo original (e.g. cambio en circunstancias de las minas cercanas, diferentes superficies de infraestructura). Escape de agua es un tema amplio que interactúa con varios sistemas de manejo, incluyendo control de suelo (superficie, subterráneo), ventilación, agua, plan de ingeniería en mantenimiento, control topográfico, inspecciones, manejo de defecto y plan de respuesta a emergencias.

- INTERFAZ HUMANO-MAQUINA hace referencia a la interfaces (e.g. controles y pantallas) que son los medios por los cuales la gente:

- Operan y mantienen plantas o equipos, ya sea fijo, portable o móviles.

- Ganar o mantener el conocimiento de la situación sobre el estado actual y potenciales futuros estados de la planta en la mina y/o relevantes aspectos de otros equipos o gente; presencia de metano, estabilidad de taludes.

- Ganar y mantener el conocimiento acerca de actuales y potenciales futuros estados de la mina y relevante ambiente (e.g. ventilación, dotación, movimientos de vehículos y maquinaria, producción, desarrollo y actividades de mantenimiento, manejo de agua, seguridad, pronostico del clima)

Interfaces de operadores incluye palancas, botones de empuje, pedales, ruedas, interruptores, pantallas táctiles, palancas de mando, válvulas, teclados, controles remoto, y dispositivos de comunicación. Interfaces de visualización incluyen fuentes de información visual (e.g. ventanas, espejos, pantallas de computador, medidores, monitores de video, lámparas de casquillo, tableros mímicos, tableros de etiquetas, señas, indicadores visuales, luces, pictogramas y tableros), también pantallas auditivas (e.g. alarmas, zumbadores, dispositivos de comunicación) y dispositivos de retroalimentación (e.g. vibrando joystick). La detección y percepción de información permite que haya una situación de conocimiento que sea permitida. La situación de conocimiento es típicamente discutida en términos de conciencia individual que puede incluir sistemas automatizados complejos y múltiples operadores. Errores en el uso de controles, u operación suboptimiza la operación de controles, puede resultar en un movimiento no intencionado o retrase la acción de la planta o un equipo que ha sido operado, permitiendo consecuencias no deseadas incluyendo lesiones, fatalidades, daño en el equipo/ incrementa mantenimiento y/o pobre rendimiento.

- HIGIENE OCUPACIONAL enfoca en agentes químicos, físicos, y biológicos. Está orientado a la minería de carbón en minas de cielo abierto, subterráneo a través de la duración de vida de la mina (exploración, operaciones, cierre). Está enfocado a principales peligros tales como polvo de carbón, polvo de sílice, material particulado de Diesel (DPM), ruido y ambiente térmico (e.g. calor, frio). Otros peligros que son incluidos como contaminantes son transportados por aire (e.g. humos, fibras, gases, vapores, productos de combustión; incluyendo el efecto de espacios confinados), contaminantes transmitidos por medio del agua (e.g. bacterias, E.coli, químicos (e.g. solventes, componentes de limpieza), radiación ionizada, no ionizada (e.g. flash de soldadura), vibración, y no aptos para la iluminación. Menos un adecuado control de estos factores de estrés en un ambiente de trabajo puede resultar en 
daños a los trabajadores. Adversos consecuencias a la salud puede sentirse inmediatamente, a corto-periodo o a largo plazo después de años de exposición (p. ejem. efectos agudos, crónicos y acumulativos, largo de periodo de latencia)

- ESCAPES DE GAS se orienta a liberación de repente de gas y material que está bajo presión en el lugar de trabajo que tiene el potencial de afectar la salud, y seguridad en la minería de carbón. Escapes de gas ocurren cuando hay suficiente volumen de gas y presión que excedan la fuerza confinada del material que ha sido minada o entre las capas de arriba y/o debajo de la capa activa. Escapes de gas generalmente ocurren en la fase de operación. Históricamente, la mayoría de los escapes de gas ocurren en la fase de desarrollo.

- LUGARES DE ALMACENAMINETO DE ESCORIA se enfoca a riesgos catastróficos de daños de represa debida a la inestabilidad geotécnica de la presa por sí misma, o por su fundación, desbordamiento, erosión de la pared de la presa o falla de la tubería de la presa. En la minería de carbón las presas de escoria también son propensos a las filtraciones a través de la pared, particularmente durante la operación cuando largas cantidades de volumen de agua son descargados en los canales. El riesgo más significante está asociado con el derrame y filtración de agua contaminada. Contaminación puede presentarse como forma elevada de salinidad, acidez y metales disueltos y sulfato. Escurrimiento de los canales de almacenamiento de la minería de carbón puede causar erosión y puede también contener contaminantes. Efluentes de agua con pequeñas piedras puedes ser liberados por la falla de presa. Tal falla puede ser por la inestabilidad de pendientes, tubería o erosión debido al desbordamiento.

El equipo de RISKGATE ensambla paneles individuales de cada tópico (equipos de expertos industriales) para discutir y desarrollar el contenido (conocimiento industrial) para cada tópico en específico. Sistemas de contenido específico fue capturado para la discusión y debate en el grupo vía a acciones semi-estructurados en ciclos de talleres de investigación descrito en mayor detalle en Kirsch et al. (2012, 2013a,b) y Worden et al. (2013). Como resultado, cada uno de las miles de causas específicas, controles y consecuencias dentro RISKGATE fue identificado, creado, evaluado y rectificado por expertos industriales de las compañías mineras más influyentes en Australia antes de cargarlo al sistema.

\section{PROGRAMA DE INVESTIGACIÓN Y PARTICIPANTES EN RISKGATE 2011-2013}

Actividades de los talleres pueden ser resumidos a continuación:

- 2011: 86 días de taller, 422 días individuales con expertos industriales en minería

- 2012: 39 días de taller, 164 días individuales con expertos industriales en minería

- 2013: 34 días de talleres, 149 días individuales con expertos de minería

Estos expertos, traen una variedad de experiencia y entrenamiento en minería subterránea, minería de cielo abierto, las canteras, ha contribuido al equivalente de 735 días individuales en el programa de RISKGATE , lo cual significa en años en la experiencia en la industria en específicos tópicos desde 10.5 años (resbalones, tropiezo, caídas) a 31.4 años (Escapes de gas) (Tabla 1). 
Tabla 1. Participación de expertos industriales en los talleres de RISKGATE

\begin{tabular}{lccc}
\hline \multicolumn{1}{c}{ Tópico } & $\begin{array}{c}\text { Número de días } \\
\text { en talleres }\end{array}$ & $\begin{array}{c}\text { Participación de talleres } \\
\text { (días de expertos } \\
\text { industriales en minería) }\end{array}$ & $\begin{array}{c}\text { Experiencia } \\
\text { de participantes } \\
\text { involucrados } \\
\text { en la realización } \\
\text { de talleres } \\
\text { (años) }\end{array}$ \\
\hline Colisiones & 11 & 51 & $24.8(1-39)$ \\
Incendios & 15 & 81 & $26.5(9-48)$ \\
Aislamiento & 15 & 83 & $19.3(14-39)$ \\
Control de suelo & 14 & 45 & $21.3(5-37)$ \\
Control de estratos de suelo & 16 & 75 & $19.4(5-37)$ \\
Neumáticos & 15 & 142 & $22.0(1-42)$ \\
Explosiones & 11 & 47 & $26.9(15-38)$ \\
Explosivos a cielo abierto & 11 & 62 & $22.9(9-40)$ \\
Explosivos en ambiente subterráneo & 3 & 14 & $20.0(5-41)$ \\
Trabajos manuales & 8 & 58 & $17.7(4-38)$ \\
Resbalones, tropiezos y caídas & 9 & 39 & $10.5(1-22)$ \\
Desastibilidad de carbón & 3 & 14 & $24.0(7-40)$ \\
Escapes de agua & 8 & 42 & $30.5(15-39)$ \\
Interface & 4 & 20 & $15.5(1-23)$ \\
Higiene ocupacional & 4 & 49 & $17.8(3-24)$ \\
Escapes de gas & 10 & 20 & $31.4(16-40)$ \\
Almacenamiento de escoria & 4 & 15 & $20.7(10-27)$ \\
\hline & & & \\
\hline
\end{tabular}

La participación de talleres está representado por una variedad de participantes representados por medio de expertos industriales en minería adquirido a través de diez compañías, catorce OEM o proveedores, tres universidades, y dos agencias regulatorias (NSW, QLD) (Tabla 2). Los esfuerzos de colaboración industrial provienen de la fundación en la que es formada RISKGATE, con resultados que reflejan como practicas líderes han sido compartidas y negociadas en la práctica actual. Integración del conocimiento intersectorial, más el apoyo de un conjunto sustantivo y diverso de la industria, por medio de recursos académicos y tecnológicos, lo que significa que RISKGATE puede ofrecer una transferencia continua de conocimiento y redefine la mayor practica en la identificación de riesgo, evaluación y en el manejo de la industria de carbón. 
Tabla 2. Participantes en los talleres 2011-2013

\begin{tabular}{|c|c|c|c|c|}
\hline Anglo American & Bridgestone & Aystar & $\begin{array}{l}\text { The University of } \\
\text { Queensland }\end{array}$ & $\begin{array}{l}\text { Qld Department } \\
\text { of Employment, }\end{array}$ \\
\hline BMA/BHP & Good Year & Dyno Nobel & & $\begin{array}{l}\text { Economic } \\
\text { Development and }\end{array}$ \\
\hline Bandana & Marathon & Golder & $\begin{array}{l}\text { University of New South } \\
\text { Wales }\end{array}$ & Innovation (DEEDI) \\
\hline Centennial Coal & Michelin & Ergo Enterprises & & \\
\hline Downer EDI & Titan & Job Fits System & University of Wollongong & $\begin{array}{l}\text { Qld Department of } \\
\text { Natural Resources } \\
\text { and Mines (DNRM) }\end{array}$ \\
\hline Gujarat & & Orica Mining Services & & \\
\hline Peabody Energy & & & & $\begin{array}{l}\text { New South Wales } \\
\text { Trade and Industry }\end{array}$ \\
\hline Rio Tinto & & Otraco & & $\begin{array}{l}\text { Workplace Health } \\
\text { and Safety }\end{array}$ \\
\hline Glencore & & Klinge Group & & \\
\hline Adani & & Pulford & & \\
\hline Caledon & & & & \\
\hline
\end{tabular}

\section{Distribución del conocimiento a través del Sistema corbatín RISKGATE}

El cuerpo de conocimiento RISKGATE usa el método BTA, en el que se enfoca al manejo de riesgo que ayuda a usuarios que puedan considerar el contexto entero de un evento no deseado asociado con un peligro en particular: sus causas, consecuencias y los más importante los controles usados para la prevención de eventos no deseados o la mitigación o reducción de consecuencias de lo que pueda ocurrir. Una ventaja clave de este método es el enfocarse en la efectividad del control. La explicación detallada de la aplicación de BTA en el programa de RISKGATE puede ser encontrada en Kirsch et al. (2012, 2013a,b) y Worden et al. (2013). Un breve resumen se presenta acá para los nuevos usuarios.

\section{Elementos Corbatín}

Hay típicamente entre cuatro y nueve corbatines en cada tópico de RISKGATE, en cada corbatín en la parte del centro está el específico evento de iniciativa (o evento no deseado). El evento de iniciativa "nudo" del corbatín representa el punto donde el control de energía se ha perdido; incluyendo las causas primarias y las consecuencias no deseadas de evento de iniciativa tabuladas en cualquiera de los lados del nudo del corbatín. Una causa es cualquier ocurrencia o razón que puede liderar a un evento que resulte en peligro(s). Correspondientemente, una consecuencia es un resultado negativo que surge de un evento de iniciativa. Las principales consecuencias de RISKGATE están asociadas con lesiones o enfermedades que afecten a personas, también incluye daños a equipos y/o al medio ambiente, que puedan representar importantes consecuencias negativas.

Controles están incluidos en todo proceso, política, práctica o cualquier otra acción que tenga la intención de reducir la probabilidad de que un evento no deseado curra o que reduzca la magnitud de las consecuencias de un evento no deseado. Las causas son prevenibles a través de controles de prevención específicos. Estos controles prevenibles podrían fallar, la severidad de las consecuencias de un evento no deseado son minimizados a través de controles de mitigación diseñados e implementados antes de que los eventos ocurran. 
La herramienta de BTA en RISKGATE tiene suficiente flexibilidad para dar cabida a un conocimiento más profundo y ser ampliado más allá de los requisitos pronosticados por el usuario, como ha sido experimentado durante el proyecto. El actual número de datos colectado a la fecha de cada evento de iniciativa por tópico esta resumido en la Tabla 3. Nota, estos controles pueden ser estimativos ya que pueden romperse en múltiples opciones o sub-controles. Los datos totales de escape de agua, Escoria e higiene ocupacional están aún en proceso de revisión.

Tabla 3. Resumen de la base de datos de elementos que conforman el Sistema corbatín en RISKGATE (un evento de iniciativa por tópico)

\begin{tabular}{lccccc}
\hline \multicolumn{1}{c}{ Tópico } & $\begin{array}{c}\text { Numero } \\
\text { de eventos } \\
\text { de iniciativa }\end{array}$ & Causas & $\begin{array}{c}\text { Controles } \\
\text { preventivos }\end{array}$ & Consecuencias & $\begin{array}{c}\text { Controles } \\
\text { de mitigación }\end{array}$ \\
\hline Neumáticos & 4 & 28 & 146 & 9 & 29 \\
Colisiones & 2 & 45 & 133 & 4 & 11 \\
Controles de estratos de suelo & 8 & 114 & 383 & 21 & 4 \\
Control de suelo & 7 & 86 & 204 & 23 & 35 \\
Incendios & 4 & 46 & 165 & 9 & 54 \\
Aislamiento & 5 & 301 & 792 & 11 & 65 \\
Explosivos en cielo abierto & 5 & 55 & 127 & 18 & 39 \\
Explosivos en entorno subterráneo & 4 & 35 & 84 & 10 & 24 \\
Explosiones & 7 & 50 & 240 & 7 & 43 \\
Trabajos manuales & 3 & 39 & 114 & 3 & 6 \\
Resbalones, Tropiezos \& Caídas & 3 & 26 & 57 & 3 & 11 \\
Bultos de carbón y erupciones & 3 & 3 & 20 & 9 & 20 \\
Escapes de gas & 1 & 7 & 31 & 1 & 6 \\
Interface & 3 & 52 & 216 & 3 & 31 \\
\hline
\end{tabular}

\section{RISKGATE: Modelos de conocimiento por tópico}

Durante los talleres de los expertos industriales encontrado en BTA han sido más efectivos cuando la estructura del modelo ha sido reconocido en la industria para asistir en un Sistema diseñado e implementado por el usuario. Los paneles de expertos en cada área del tópico escogió diferente modelo o estructura para la adquisición de conocimiento en la industria y la presentación en el sistema en línea. Esta tendencia coincidió con los enfoques únicos que los expertos en minería utilizan en el "sitio" para gestionar su riesgo particular. Por ejemplo, los tópicos de incendios y explosiones fueron estructurados en base a las fuentes de combustibles o ignición, con las fuentes de combustión del tópico de incendio dividido en líquidos inflamables; carbón; otros solidos; gases presurizados; y otros fluidos inflamables. Los estratos de suelo (minería subterránea) y suelo (minería de cielo abierto) fueron divididos de acuerdo a los eventos no deseados en diferentes locaciones de minería, y controles de cada uno de estas categorías de acuerdo si se enfocan en el diseño o los problemas operacionales. La información del tópico de neumáticos fue desarrollada alrededor del ciclo de vida del neumático desde su compra hasta su disposición. El tópico de aislamiento fue posible realizar una matriz compleja de 5x4 entre la "vida de los activos" (diseño/compra; instalación/puesta en marcha/ modificación; y cierre) y los pasos de aislamiento (identificación; operación, verificación; y garantía). Estos modelos o estructuras fueron usados a través de la acción de investigación en los talleres para asegurar que los peligros identificados sean enviados de una manera comprensiva y sistemática. 


\section{RISKGATE: operaciones integradas en la compañía}

Los 11 primeros tópicos de RISKGATE fueron lanzados en Diciembre 2012, con el anuncio de seis adicionales tópicos que venían en camino a finales de Diciembre del 2013. Por lo tanto la transferencia de tecnología e implementación del sistema esta aun en una fase temprana en la industria de carbón en Australia. Sin embargo, los siguientes casos de estudio proveen ejemplos de cómo RISKGATE está siendo usado en la actualidad en diferentes compañías mineras de carbón:

\section{Uso de RISKGATE como referencia/cuerpo de conocimiento}

El punto de entrada para un usuario nuevo en RISKGATE es la familiarización con la estructura del sitio, después de descargar la información en la forma de tener acceso a las listas de verificación personalizadas pueden ser usadas para informar a la corporación o evaluaciones de riesgo a nivel de sitio, incidentes de investigación, auditores y para el desarrollo del manejo de sistemas. Por ejemplo, Peabody Energy Australia está usando la información de los estratos de suelo y suelo de los tópicos de RISKGATE como material de referencia para auditores en el plan de manejo de peligros geológicos/geotécnicos en operaciones específicos

\section{Consideraciones de contenido de RISKGATE en el desarrollo de prácticas corporativas}

Una vez que el sistema es familiar, y su detallado cuerpo de conocimiento, las compañías están empezando a usar la información de RISKGATE como base de datos comparativa para el análisis de estándares corporativos y el informe de desarrollo de nuevos estándares o como una práctica recomendada. Anglo American ha usado RISKGATE como punto de referencia en sus estándares en el tópico de aislamiento a nivel global; y su nueva recomendación para el sistema está en la reducción de colisiones tomando como base el conocimiento de colisiones de RISKGATE como primera fuente.

\section{Integración del contenido de RISKGATE en los sistemas de operación}

Algunas compañías de carbón están integradas activas a RISKGATE en el proceso de manejo de riesgos en sus procesos. En la vanguardia de estos esta Centennial Coal, que abarca completamente el uso de RISKGATE y en el desarrollo de un interfaz de software que permita la integración de la base de conocimiento de RISKGATE dentro del manejo interno de riesgo en el sistema de software, Stature. El personal de Centennial puede entrar a la página web de RISKGATE, personalizar las listas de verificación para su aplicación específica, guarda estas listas de chequeo en el formato de Stature en el ordenador central; y luego carga la carpeta de RISKGATE para que aparezca en la página de Stature.

Cuando esto ha sido completado, el gestor de riesgos es capaz de retornar a los procedimientos estándares y de rutina para el manejo de riegos, pero ahora están equipados con el cuerpo de conocimiento del riesgo en la industria de carbón en Australia. Esto es como traer todo el proceso de la industria a un sitio al mismo tiempo. Este proceso ha sido particularmente beneficioso en la evaluación de riesgos, donde los usuarios puedan compilar la colección de sus propios controles con los de RISKGATE enfocándose en particular en un evento no deseado o peligro, y este pueda traer información a un grupo más amplio que conduzca a la evaluación de riesgo

\section{RISKGATE: Futuros pasos y futuras oportunidades}

En el 2014, RISKGATE se enfocara en el tópico que queda Salud ocupacional, pendiente de la respuesta de fondos de ACARP. Esta propuesta de este tópico se ha dividido en cinco áreas principales que se dirija al consumo de drogas (legal e ilegal), alcohol, fatiga, bienestar físico y bienestar sicológico. En agosto de 2013, el equipo de RISKGATE hizo 106 encuestas a industrias mineras representativas en Australia para la 
ayuda del equipo en el desarrollo del proyecto de salud ocupacional y obtener nuevas aportaciones en los talleres de acción de investigación. Las encuestas mostraron un fuerte soporte en las cinco categorías del tópico mencionado y los encuestados contribuyeron con sugerencias sobre lo que podría incluirse dentro de estas categorías. Estos incluyen:

- Cafeína, sintéticos y el impacto en las familias - consumo de drogas

- Comprensión de que es un consumo "normal" - alcohol

- Frecuentes viajes, cuidado personal, auto-control, rendimiento, salud personal, alcohol, calidad de dormir -fatiga

- Manejo de dolor, desordenes de sueño, cuidado personal, requisitos de aptitud física en casos de emergencia, condiciones musculo-esquelético- bienestar físico.

- Impactos de medicamentos, técnicas de evaluación, soporte familiar - bienestar sicológico.

El equipo de RISKGATE está buscando expertos industriales quienes puedan contribuir su conocimiento en el tópico de Salud ocupacional 2014 a través de talleres de acción investigativa. Los talleres de participación representan oportunidades para la creación de contactos, compartir y reflexionar de lecciones aprendidas, mantenerse al corriente de controles emergentes en tecnología, cambio en la cultura de seguridad existente, y elevar niveles de practica actuales.

Sin embargo, el cuerpo de conocimiento ha sido aplicado más allá de la industria carbonífera. Muchos de los peligros en la industria de carbón, tales como colisiones, energía riesgosa (por ejemplo electricidad o presión hidráulica), incendios, explosiones y resbalones/tropiezos/caídas son muy comunes en otros dominios mineros y en otras industrias. La minería carbonífera es reconocida globalmente como una actividad riesgosa y, como resultado, operan bajo estrictas niveles de regulación y escrutinio público. Otras de las industrias de alto riesgo, la mayoría asociadas con el suplemento de carbón o cadenas de energía -incluyendo generación de energía y transmisión, construcción, transporte ferrocarril, carreteras y embarque- y otras industrias mineras también necesitan el manejo de fuerzas de trabajo que operan en entornos similares de alto riesgo.

Desde una amplia perspectiva industrial, la plataforma de RISKGATE contribuye en la captura de conocimiento y el intercambio de conocimiento con respecto a las prácticas actuales, y las facilidades del establecimiento de la memoria acumulativa corporativa. Practicantes de otras riesgosas industrias son motivadas a comprometerse al proceso de RISKGATE para ayudar a mejorar los resultados obtenidos en el manejo de riesgos.

\section{AGRADECIMIENTOS}

Nosotros agradecemos a la administración de RISKGATE, diseño y soporte de comunicación (Barbara Whittaker, Sandy Worden) y los líderes de los tópicos (Bruce Hebblewhite, Guldidar V. Kizil, Tilman Rashe, Alastair Torrance, Duncan Chalmers, David Williams, Robin Burgess-Limerick, Mark Spinks, Jim Galvin, Peter Bergin). Este proyecto esta soportado a través de la concesión ACARP no. C20003. Nosotros especialmente agradecemos a cada una de los profesionales de las industrias mineras de carbón de Australia quienes dieron generosamente su tiempo y conocimiento en cada uno de los tópicos de RISKGATE, y la expresión de agradecimiento a cada una de las compañías que soportaron en la contribución de expertos y recursos técnicos. Finalmente, nosotros agradecemos a las contribuciones de revisores anónimos para la finalización de este manuscrito. 


\section{REFERENCIAS BIBLIOGRÁFICAS}

1. Harris, J., Kirsch, P., Sprott, D., Spinks, M., Goater, S. and Cliff, D., 2012. RISKGATE - a case study in application to fires on mobile plant. Proceedings of the Eighth AUSIMM Open Pit Operators Conference, Perth, 18-19 September 2012, Perth, Australia. The Australasian Institute of Mining and Metallurgy (The AusIMM).

2. Harris, J., Sprott, D., Torrance, A., Shi, M, Ranjan, A., Sharma, S., Biswas, T., Sharma, S. and Kirsch, P A, 2013. Sharing industry knowledge to improve management of risks and safety in the use of explosives in surface mining. Proceedings of the World Mining Congress, Montreal, Canada, August 11-15, 2013.

3. Kirsch, P., Goater, S., Harris, J., Sprott, D., and Joy, J., 2012. RISKGATE: Promoting and redefining best practice for risk management in the Australian coal industry. Proceedings of the 12th Coal Operators Conference, University of Wollongong \& The Australasian Institute of Mining and Metallurgy, 2012, 315-325.

4. Kirsch, P., Harris, J., and Sprott, D., 2013a. Vertical integration of risk management in the Hunter Valley Coal Chain - application of the coal industry's RISKGATE platform. The AUSIMM Bulletin February 2013, 45-49.

5. Kirsch, P. A., Harris, J., Cliff, D. and Sprott, D, 2013b. Industry scale knowledge management - RISKGATE and Australian coal operations. Proceedings of the World Mining Congress, Montreal, Canada, August 11-15, 2013.

6. Kirsch, P A, Harris, J, Cliff, D, Hebblewhite, B, Sprott, D, Shi, M, Ranjan, A, Sharma, S, Biswas, T, and Sharma, S, 2013c. Industry scale knowledge management - introducing the RISKGATE Underground Strata and Explosions Body of Knowledge. Proceedings of the World Mining Congress, Montreal, Canada, August 11-15, 2013.

7. Kirsch, P., Harris, J., Sprott, D, and Cliff, D, 2014. RISKGATE and Australian coal operations. Proceedings of the 14th Coal Operators' Conference, University of Wollongong \& The Australasian Institute of Mining and Metallurgy, 2014 (this volume).

8. Lynas, D., Burgess-Limerick, R., and Kirsch, P A, 2014 (in press). RISKGATE analysis of Slips, Trips and Falls at NSW surface and underground coal mines. Journal of Health $\&$ Safety Research \& Practice.

9. RISKGATE, 2013. www.riskgate.org.

10. Worden, S., Sprott, D., and Whittaker, B., 2013. Risk management competence in Australia gets a boost from new software. Mining Engineering 65 (July): 22-33. 